\title{
Clinical potential, safety, and tolerability of arbaclofen in the treatment of autism spectrum disorder
}

This article was published in the following Dove Press journal:

Drug, Healthcare and Patient Safety

10 May 2014

Number of times this article has been viewed

\section{Richard E Frye}

Arkansas Children's Hospital Research Institute, Department of Pediatrics, University of Arkansas for Medical Sciences, Little Rock, AR, USA
Abstract: Autism spectrum disorder (ASD) is a behaviorally defined disorder which has increased in prevalence over the last two decades. Despite decades of research, no effective treatment is currently available. Animal models, as well as other lines of evidence, point to abnormalities in the balance of cortical excitation to inhibition in individuals with ASD, with this imbalance resulting in an overall increase in cortical excitation. To reduce cortical excitatory glutamate pathways, arbaclofen, a selective agonist of the gamma aminobutyric acid receptor type B, has been developed. This article reviews the evidence for this treatment for ASD using a systematic review methodology. Overall, a systematic search of the literature revealed 148 relevant references with the majority of these being review papers or news items that mentioned the potential promise of arbaclofen. Five original studies were identified, four of which used STX209, a form of arbaclofen developed by Seaside Therapeutics, Inc., and one which used R-baclofen. In an animal model, treatment of Fragile X, a genetic disease with ASD features, demonstrated a reversal of behavioral, neurological, and neuropathological features associated with the disease. One double-blind, placebo-controlled study treated children and adults with Fragile X. Results from this study were promising, with signs of improvement in social function, especially in the most severely socially impaired. Two studies, one open-label and one double-blind, placebocontrolled, were conducted in children, adolescents, and young adults with ASD. These studies suggested some improvements in socialization, although the effects were limited and may have been driven by individuals with ASD that were higher-functioning. These studies and others that have used arbaclofen for the treatment of gastroesophageal reflux suggest that arbaclofen is safe and well-tolerated. Clearly, further clinical studies are needed in order to refine the symptoms and characteristics of children with ASD that are best treated with arbaclofen.

Keywords: autism spectrum disorder, Fragile X, gamma-aminobutyric acid, arbaclofen, R-baclofen, STX209

\section{Introduction}

The autism spectrum disorders (ASD) are a group of behaviorally defined neurodevelopmental disorders that have potentially life-long consequences. They are defined by impairments in communication and social interaction, along with restrictive and repetitive behaviors. ${ }^{1}$ The definition of ASD has recently undergone revision due to the difficulty with subtyping ASD. Previously, The Diagnostic and Statistical Manual of Mental Disorders (DSM) Version IV Text Revision divided ASD into several diagnoses with the most prevalent diagnoses being autistic disorder, Asperger syndrome, and pervasive developmental disorder-not otherwise specified. However, the current revision of the DSM, the DSM version 5, does not differentiate between these subtypes of ASD and considers communication and social impairments together, rather than separate symptom
Correspondence: Richard E Frye Arkansas Children's Hospital Research Institute, Slot 5 I2-4IB, 13 Children's Way, Little Rock, AR, USA

$\mathrm{Tel}+\mathrm{I} 50 \mathrm{I} 3644662$

Fax + I $50 \mid 9786483$

Email refrye@uams.edu 
classes. ${ }^{2}$ It is believed that this change may decrease the overall prevalence of ASD. ${ }^{3}$ However, most research has used the former version of the DSM to define ASD, and currently, ASD has been estimated to affect one out of 68 individuals in the United States of America, ${ }^{4}$ with four times more males than females being affected. ${ }^{5}$ Over the past two decades, the incidence of the ASDs has grown dramatically, although the reasons for this increase are continually debated.

Despite decades of research, the etiology of ASD is unclear at this time. Several genetic syndromes, such as Fragile X syndrome (FXS) and Rett syndrome, have been associated with ASD, yet empirical studies have estimated that single gene and chromosomal defects only account for a minority of ASD cases. ${ }^{6}$ Evidence has accumulated that implicates a role of environmental factors in ASD. ${ }^{7}$ For example, one recent study of 192 twin pairs reported that environmental factors were estimated to account for $55 \%$ of the risk of developing autistic disorder compared with $37 \%$ for genetic factors; a similar risk pattern was also observed for developing the broader diagnosis of ASD. ${ }^{8}$

Despite a better understanding of some of the genetic, environmental, and physiologic abnormalities associated with ASD, a better understanding of how these factors influence brain function and development is still lacking. ${ }^{9}$ One of the more promising avenues of research has converged on the excitatory-inhibitory balance of cerebral cortical circuits. Indeed, ASD may be associated with cortical hyperexcitability, due to deficits in cortical inhibitory circuits or glutamate receptor pathway abnormalities..$^{10-12}$

Some of the strongest evidence of excitatory-inhibitory imbalances in the brains of individuals with ASD is derived from the Fmr1-knockout mouse. This is an animal model of FXS, the most prevalent single gene disorder in the ASD population, which accounts for approximately $1 \%-5 \%$ of boys with ASD. ${ }^{7}$ FXS includes many cognitive and behavioral deficits that are seen as comorbid conditions in ASD, including intellectual disability, epilepsy, anxiety, aggression, and attention deficit hyperactivity disorder, ${ }^{13}$ and approximately $20 \%$ of boys with FXS meet the criteria for ASD. ${ }^{14}$ FXS is a trinucleotide repeat disorder that results from a failure to transcribe the FMR1 gene, a gene that encodes the FXS mental retardation protein. This protein is essential for regulating protein synthesis, particularly at excitatory synapses, and is intimately involved in neural plasticity. The Fmr1-knockout mouse model shows similar neuropathology to that found in the human brain of FXS boys, including increased dendritic spine density and excessive protein synthesis.
The Fmr1-knockout mouse model has provided insights into the neuropathology of FXS and ASD, particularly the abnormalities in the excitatory-inhibitory balance associated with ASD. Specifically, studies have demonstrated that mGluR1 and mGluR5, two metabotropic excitatory glutamate receptors, when stimulated by glutamate, promote protein synthesis, thereby worsening the already ongoing excessive protein synthesis at excitatory synapses. ${ }^{15}$ Studies have demonstrated that reducing mGluR5 stimulation results in a reduction in the abnormal behavior characteristic of the Fmr1-knockout mouse model. ${ }^{16,17}$ Two avenues have been developed to address this abnormality in the balance of excitatory-inhibitory neurotransmission. First is the development of glutamate antagonists ${ }^{16,18}$ in order to reduce cortical excitation. The second is to increase neural inhibition. The major cortical inhibitory pathway relies on gamma-aminobutyric acid (GABA) signaling. GABA $_{B}$ agonists like STX209 ${ }^{19}$ are particularly interesting as they act both pre- and post-synaptically. Pre-synaptically, GABA $_{B}$ agonists can actually inhibit the release of glutamate into the synaptic cleft.

For several decades, baclofen, a GABA receptor type B agonist, has been utilized both orally and intrathecally to treat spasticity by increasing the GABAergic pathways that participate in the monosynaptic and polysynaptic spinal reflex loop. Oral baclofen, which is the racemic mixture of the $\mathrm{R}$ and $\mathrm{S}$ isomers, can have significant adverse effects. In particular, baclofen, especially at high doses, tends to cause somnolence. ${ }^{20,21}$ Several forms of baclofen have been recently developed to improve its tolerability. Arbaclofen is the active R-enantiomer of baclofen (Figure 1). STX209, developed by Seaside Therapeutics, Inc. (Cambridge, MA, USA), has been used in clinical studies investigating treatments for FXS and ASD. The pharmacodynamics and metabolites of arbaclofen are slightly different than racemic baclofen. This is because only the S-enantiomer produces a metabolic byproduct. ${ }^{20}$ Thus, the R-enantiomer does not have a metabolic byproduct, hastening its elimination and simplifying the pharmacodynamics of its systemic and central effects.

Arbaclofen has been developed for several other conditions besides ASD and FXS. For example, arbaclofen is being used in ongoing clinical trials to treat spasticity in multiple sclerosis. Arbaclofen placarbil has been developed for gastroesophageal reflux. It is a prodrug with significantly superior absorption as compared to baclofen, thus improving the stability of blood levels. ${ }^{21}$ Several double-blind, placebo-controlled (DBPC) clinical trials have demonstrated 


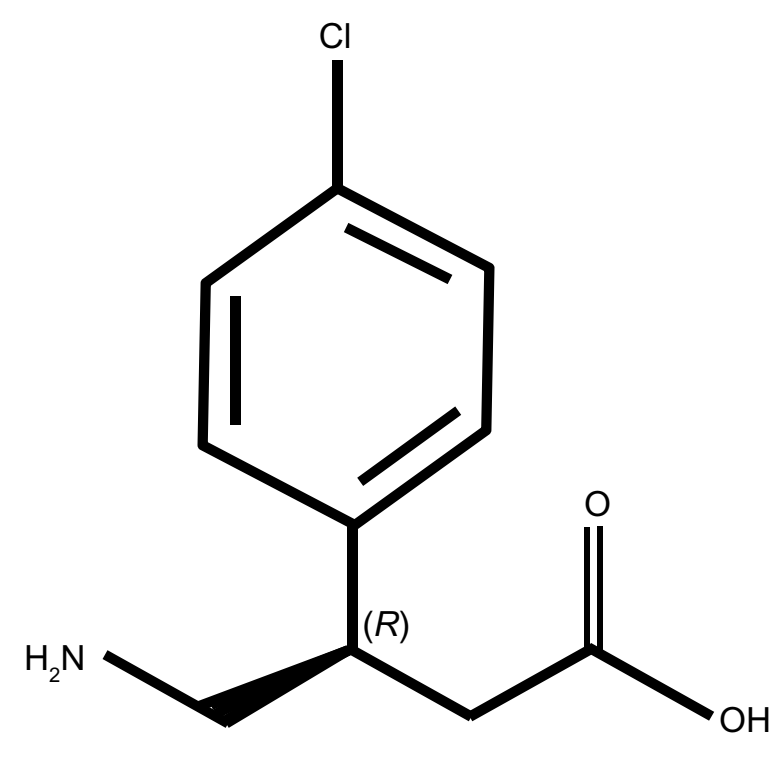

R-baclofen

Figure I The molecular structure of R-baclofen.

some efficacy of arbaclofen placarbil for gastroesophageal reflux in specific subgroups of patients. ${ }^{22-24}$ Arbaclofen placarbil has also been shown to demonstrate efficacy for spasticity in patients with spinal cord injuries in a DBPC crossover study. ${ }^{25}$

Since this is a promising treatment for these neurodevelopmental disorders that have no alternative US Food and Drug Administration (FDA)-approved medical treatment for the symptoms that define the disorders, it is important to understand the evidence for this novel treatment. Thus, this review systematically examines the literature on the use of arbaclofen for treating ASD and related disorders, reviewing both the animal and human studies that provide support for its use as a novel treatment.

\section{Methodology}

The PICO (patient problem or population [P], intervention [I], comparison [C], and outcome(s) [O]) framework was used to conduct this review. The goal was to find research studies that address the use of arbaclofen in patients or models of ASD. Arbaclofen was not compared to any other treatment and all study designs were considered. The primary goal was to consider improvement in any symptom or pathological marker associated with ASD as a result of arbaclofen treatment.

A prospective protocol for this systematic review was developed a priori, and the search terms and selection criteria were chosen in an attempt to capture all pertinent publications. A computer-aided search of PubMed, Google Scholar, CINAHL, Embase, Scopus, and ERIC databases from inception through to February 2014 was conducted to identify pertinent publications using the search terms "autism", "autistic", "Asperger", "ASD", "pervasive", and "PDD" in combination with the term "arbaclofen" or "R-baclofen". The references cited in identified publications were also searched to locate additional studies. Studies were screened by reviewing abstracts of all potentially relevant publications. Studies were included if they: 1) involved individuals or animal models of ASD and 2) reported arbaclofen as an intervention. Articles were excluded if: 1) they were conference proceedings without a published abstract, 2) did not present new or unique data, or 3) presented duplicate data.

An analysis of the level of evidence for the treatment of arbaclofen for ASD was also provided in order to quantitatively analyze the strength of evidence for this treatment. The strengths and weaknesses of the included studies were ranked using a well-established level of evidence scale. ${ }^{26}$ Using this scale, each study was individually assessed to determine the corresponding level of evidence, ranging from level 1 to 5 (Table 1). After assessing all identified studies for each treatment, a grade of recommendation was derived for arbaclofen (Table 2) ranging from A (solid evidence) to D (troublingly, inconsistent, or inconclusive evidence). We summarized and synthesized the information about arbaclofen regarding its efficacy but also then reviewed the evidence for its tolerability and safety. Finally, we provided recommendations for further studies.

\section{Results and discussion Literature related to arbaclofen}

Figure 2 provides the results of the systematic review, which revealed 148 unique, relevant articles concerning arbaclofen and ASD, with the majority of these articles being review articles or news articles. Only nine articles were original

Table I Levels of evidence

\begin{tabular}{ll}
\hline Level & Description \\
\hline Ia & SR or meta-analysis of RCTs with homogeneity or Cochrane \\
& review with favorable findings \\
Ib & Prospective high-quality RCT \\
$2 \mathrm{a}$ & SR of cohort (prospective, nonrandomized) studies with \\
& homogeneity \\
$2 b$ & Individual cohort (prospective, nonrandomized) study or \\
& low-quality RCT \\
$3 \mathrm{a}$ & SR of case-control (retrospective) studies with homogeneity \\
4 & Individual case-control (retrospective) study \\
5 & Case series or reports \\
& Expert opinion without critical appraisal or based on \\
\hline
\end{tabular}

Abbreviations: RCT, randomized controlled trial; SR, systematic review. 
Table 2 Grade of recommendation

\begin{tabular}{ll}
\hline Grade & Description \\
\hline A & At least one level Ia study; or two level Ib studies \\
B & At least one level Ib, 2a, or 3a study; or two level $2 \mathrm{~b}$ or \\
& 3b studies \\
C & At least one level 2b or 3b study; or two level 4 studies \\
D & Level 5 evidence; or troublingly inconsistent or inconclusive \\
& studies of any level; or studies reporting no improvements \\
$\mathrm{N}$ & No studies identified \\
\hline
\end{tabular}

research reports, with four of these reports specifically concerning ASD, with the other five reports considering FXS, which is regarded as a model of ASD. For the reports on FXS, one published paper concerned human participants, while two published papers concerned animal models. The other two studies were meeting abstracts that reported the same data as the published papers. For the reports on ASD, there was only one published paper on human participants, while the other four reports were meeting abstracts. One meeting abstract reported original data from a clinical trial while the others reiterated the data from the published paper.

\section{Evidence from animal models}

The Fmr1-knockout mouse model shows similar neuropathology to that found in the human brain of FXS boys and is a model of ASD (as described in the Introduction). To determine if STX209 could provide improvement in both behavioral and neuropathological characteristics of the Fmr1-knockout mouse, age-matched male wild-type and Fmr1-knockout littermates with and without STX209 treatment where compared..$^{27}$ Neuropathological measurements included protein synthesis and $\alpha$-amino-3-hydroxy-5-methyl4-isoxazolepropionic acid (AMPA) receptor trafficking in hippocampal neurons, polysome profiling in brain tissue, and spine density in pyramidal neurons from the binocular visual cortex. Behavioral measures included the incidence of audiogenic seizures, marble burying (a measure of repetitive behavior) and the open field traveling test (an index of general motor activity).

Overall, this study demonstrated that STX209 reversed neuropathological and behavioral abnormalities in the Fmr1knockout mouse. Treatment with STX209 decreased protein synthesis and AMPA receptor internalization in hippocampal neurons, increased the $80 \mathrm{~S}$ monosome to polysome ratio (an indication of decreased protein translation), and corrected the excess dendritic spine density characteristic of the Fmr1-knockout mouse. STX209 treatment also corrected behavioral abnormalities associated with the Fmr1-knockout mouse. STX209 significantly reduced the incidence of audiogenic seizures in the Fmr1-knockout mouse, with a significantly lower minimum effective dose as compared to racemic baclofen. STX209 also significantly reduced marble burying, a measure of repetitive behavior, in the Fmr1-knockout

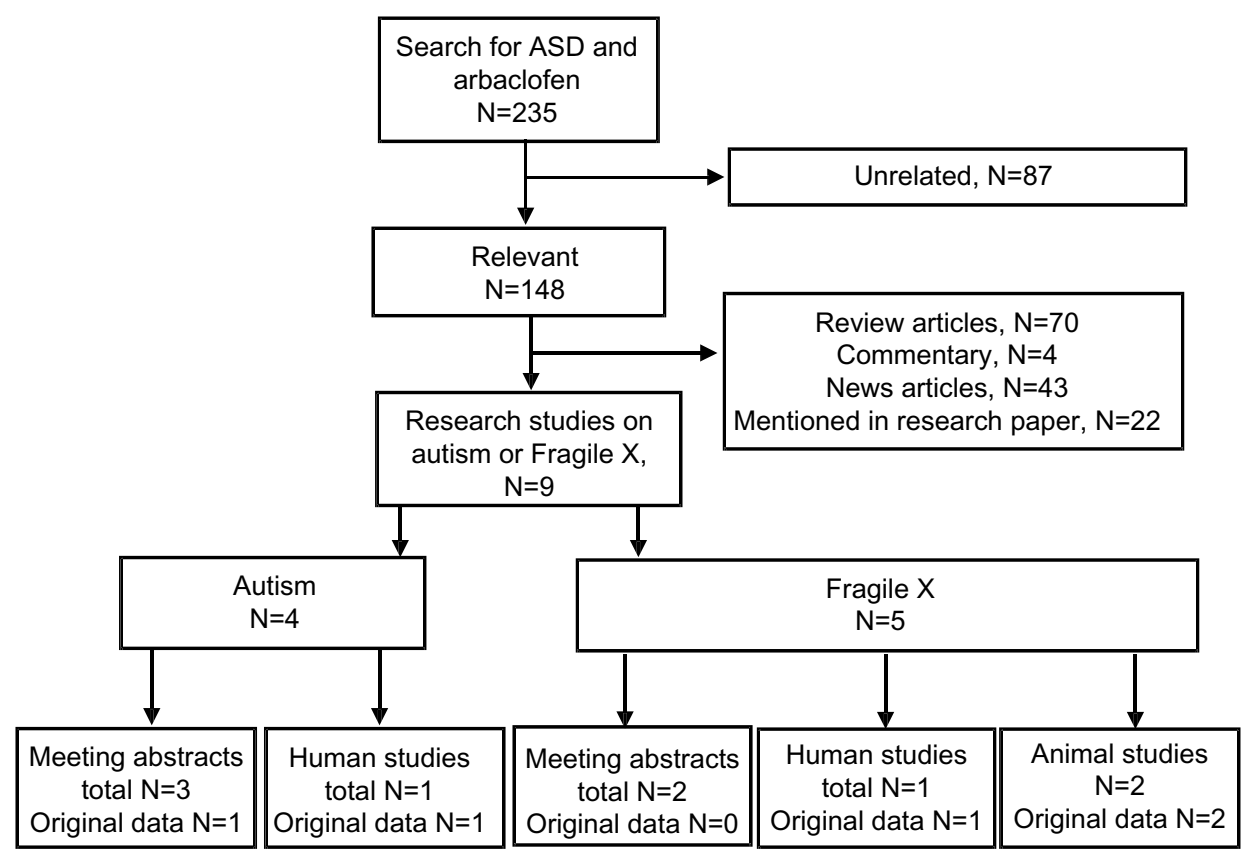

Figure 2 Flow diagram of systematic literature search for articles concerning autism (including Fragile X Syndrome) and arbaclofen or R-baclofen.

Notes: The last boxes outline the total number of studies published that present research data and define whether the reports discuss data that are original or a discussion of data reported elsewhere (ie, duplication data). Duplicate data was exclusively reported in abstract form.

Abbreviation: ASD, autism spectrum disorder. 
mouse. However, since STX209 also reduced locomotion in the open field traveling test, the authors indicated that such reduction in locomotion could have accounted for some or all of the effect of the marble burying test. With this positive behavioral and physiological data, clinical trials were initiated for STX209.

It should be mentioned that a previous study demonstrated that R-baclofen significantly reduced audiogenic seizures in the Fmr1-knockout mouse with a single dose of $1 \mathrm{mg} / \mathrm{kg}$, but not when provided subchronically for 6 days. ${ }^{28}$ In this study, $\mathrm{R}$-baclofen was also found to reduce audiogenic seizures in the Fmr1-knockout mouse when provided at a lower dose $(0.5 \mathrm{mg} / \mathrm{kg})$ along with the mGluR5 inverse agonist 2-methyl6-(phenylethynyl) pyridine. ${ }^{28}$ This study also demonstrated that R-baclofen did not significantly change the expression of $\mathrm{GABA}_{\mathrm{B}}$ receptors. ${ }^{28}$

\section{Evidence from human studies}

Human studies have been conducted with STX209 on both ASD and FXS populations. For the FXS population, one multisite randomized DBPC crossover study with washout was performed in the US over 15 months on 63 adults (up to 39 years old) and children (as young as 6 years old). ${ }^{29}$ Dosing was flexible, with tapering up of the drug every 3-4 days to a maximum of $10 \mathrm{mg}$ twice a day for children and $10 \mathrm{mg}$ three times per day for adults until the optimal tolerated dose was established. The participants were evaluated at 2 and 4 weeks after starting the treatment and then tapered off the treatment over 1-2 weeks. A 7-day washout period separated the two treatments (placebo and STX209). Assessment measures included the Clinical Global Impression Scale (CGIS), the Aberrant Behavior Checklist (ABC), Vineland Adaptive Behavior Scales (Second Edition; VABS), the Social Responsiveness Scale (SRS), the Repetitive Behavior Scale-Revised, the Child and Adolescent Symptom Inventory Anxiety Scale, the Attention Deficit Hyperactivity Disorder Rating Scale-IV, a measure of vocabulary and short-term and working memory, and a Visual Analog Scale (VAS) of the child's three most problematic behaviors. Overall, the primary endpoint, the irritability subscale of the $\mathrm{ABC}$, was not significantly different between active treatment and placebo portions of the trial but a secondary endpoint, the VAS problem behavior rating, was significantly better with the active treatment as compared to the placebo. Another secondary measure, the social avoidance ABC subscale, was found to significantly improve more during active treatment as compared to placebo. In addition, when the FXS participants with the worst social impairments were analyzed separately, multiple outcome measures, including the VABS socialization subscale, the CGIS Severity and Improvement, clinical and parent treatment preference, and percentage of responders, were significantly better during active treatment as compared to placebo. Thus, overall, this study supports the notion that STX209 improved social function, particularly in FXS individuals with the most significant social impairments. This study did suffer from some significant limitations, including participant heterogeneity and the relatively short exposure to active treatment, especially considering the fact that the drug dose was being optimized during the relatively short treatment period.

Two clinical studies, one open-label and one DBPC, ${ }^{30,31}$ have been conducted in individuals with ASD using STX209. In an 8-week, open-label trial, 32 children and adolescents with $\mathrm{ASD}$ and an $\mathrm{ABC}$ irritability score $\geq 17$ were titrated up to optimal tolerated dose, increasing the dose every 3-4 days to a maximum dose of $10 \mathrm{mg}$ twice a day for participants aged 6-11 years of age or $10 \mathrm{mg}$ thrice a day for participants aged $12-17$ years of age. ${ }^{30}$ Outcome measures including the $\mathrm{ABC}$, the CGIS Severity and Improvement, the SRS, the Children's Yale-Brown Obsessive Compulsive Scale - modified for Pervasive Development Disorder, the Child and Adolescent Symptom Inventory-4 Anxiety scale, the Attention Deficit Hyperactivity Disorder Rating Scale$\mathrm{IV}$, the VABS, and the Leiter-R Brief intellectual quotient, occurred every 2 weeks. Significant improvement occurred on all primary and secondary outcomes measures except the VABS, the Leiter-R Brief intellectual quotient, and the ABC inappropriate speech subscale. Overall, $41 \%$ were rated as responders using a very rigorous criterion. This initial openlabel study confirmed the tolerability of STX209 in the ASD population and demonstrated possible efficacy for a broad range of outcome measures, suggesting that further controlled studies were warranted.

To further investigate the efficacy of STX209 in the ASD population, a 12-week DBPC trial was conducted on 150 children, adolescents, and young adults with ASD and reduced social function (as defined by an ABC lethargy/social withdrawal subscale $\geq 8$ ). ${ }^{31}$ Treatment was titrated over 4 weeks to a maximum of $10 \mathrm{mg}$ thrice a day for participants aged 5-11 years of age or $15 \mathrm{mg}$ thrice a day for participants aged 12-21 years of age, with 130 completing the study. Outcome measures included the ABC, the CGIS Severity and Improvement, and the Socialization and Communication subscales of the VABS. The primary outcome measure, the ABC lethargy/ social withdrawal subscale, was not significantly different between the STX209 and placebo groups. For the secondary 
outcomes measures, CGIS Severity significantly improved more in the STX209 group as compared to the placebo group, but other secondary endpoints did not demonstrate greater improvements in the STX209 group as compared to the placebo group. However, a per protocol analysis demonstrated a significant improvement in the VABS socialization subscale in the STX209 as compared to the placebo group. The improvement in the VABS socialization subscale was also more evident in higher functioning participants. This study demonstrated that STX209 improved a measure of social function in some children with ASD but that future studies are needed to define the precise characteristics of the individuals with ASD that will optimally respond to STX209. Since this study has only been reported in abstract form, it is difficult to assess some of the potential limitations and the significance of the subgroup analyses.

\section{Grade of recommendation}

For FXS, there are two bench research studies ${ }^{27,28}$ (Level 5) and one high-quality randomized controlled study ${ }^{29}$ (Level 1b), resulting in a Grade of Recommendation of B for improving social function in individuals with FXS. This needs to be tempered by the fact that the evidence supports the effect of STX209 for a subgroup of FXS patients with poor social function. For the ASD population, there is one open-label trial $^{30}$ (Level 2b) and one high-quality randomized DBPC trial $^{31}$ (Level 1b), resulting in a Grade of Recommendation for individuals with ASD of B for improving social function. This recommendation does, however, need to be tempered by the fact that STX209 appears to have an effect on the subgroup of ASD children who are high-functioning and that the participants in the clinical trials were preselected to be irritable in one study ${ }^{30}$ (the open-label study) and to have poor social function in another ${ }^{31}$ (the DBPC study).

\section{Safety and tolerability}

Overall, the clinical studies suggest that STX209 is well-tolerated. In the FXS DBPC trial, the incidence of adverse events was not significantly different between the active treatment and placebo arms of the study, although the one serious adverse event that occurred was in the active treatment arm. The individual was hospitalized for increased irritability; the individual had a history of previous hospitalizations for similar behavioral issues. ${ }^{29}$ In the openlabel ASD trial, STX209 was well-tolerated, with the most common adverse events being agitation, irritability, fatigue, hyperactivity, insomnia, and diarrhea, with the majority of the adverse events rated as mild and resolving without changing the dose. ${ }^{30}$ In the DBPC study on individuals with ASD, approximately $11 \%$ of participants on STX209 discontinued because of adverse events, with the majority of the adverse events being behavioral. ${ }^{31}$ This is compared to approximately $3 \%$ of participants on placebo. There was only one serious adverse event on STX209, suicidal ideation, which also occurred in one placebo participant. Thus, in the three STX209 clinical studies conducted on individuals with ASD or FXS, STX209 was well-tolerated with a low incidence of adverse events.

Clinical studies ${ }^{22-25}$ in other disease populations have used arbaclofen placarbil, a non-STX209 form of arbaclofen. Although this form of arbaclofen has slightly different pharmacodynamics than STX209, some safety data can be obtained from these other clinical studies. In three DBPC trials for gastoesophegeal reflux, arbaclofen placarbil was found to be generally well-tolerated without a significant increase in adverse events in the treatment groups related to the medication. ${ }^{22-24}$ The most common adverse events in these studies were nausea, somnolence, dizziness, and headache, with one study reporting a dose-related increase in these adverse events.

\section{Current product development}

One of the primary goals of Seaside Therapeutics, Inc., was to develop targeted treatments for developmental disorders such as FXS and ASD based on modulation of the excitatoryinhibitory imbalance in neuronal pathways. As part of this venture, Seaside Therapeutics, Inc. developed several compounds, including STX209 and STX107 which is a selective mGlu5 antagonist. STX209 was the flagship compound that was used in several clinical trials in FXS and ASD patients. In June 2012, Seaside Therapeutics, Inc. announced a partnership with the giant pharmaceutical company Roche to further develop its compound. However, when the primary clinical outcome endpoint of the recent STX209 clinical trials did not attain statistical significance Roche backed out of its partnership with Seaside Therapeutics, Inc. Coincident with this announcement, Seaside Therapeutics, Inc. prematurely closed STX209 open-label extension studies for patients with FXS and ASD, citing limited resources to continue clinical trials. The former vice president of Seaside Therapeutics, Inc., Dr Paul Wang, has been quoted several times emphasizing that STX209 did demonstrate significance at several secondary endpoints in the clinical studies. Despite this positive outlook, Dr Wang left the company and is now the Senior Vice President and Head of Medical Research at Autism Speaks. STX209 does not appear to be available to patients on a clinical or research basis, even for compassionate use. 


\section{Future research}

Clearly, preliminary clinical studies support the notion that arbaclofen in the form of STX209 has promise for improving social function in the ASD population. However, it is clear that it is more effective in particular subpopulations of individuals with ASD. Although the limited studies have provided some mixed results, it is clear that they point to social function as the target outcome measure and indicate that arbaclofen may be most effective for individuals with ASD that have particular characteristics. Thus, future clinical studies will need to carefully select participants or be designed to account for the known heterogeneity in the ASD population. Given the lack of treatment for children with ASD and FXS, hopefully a commercial or research organization will obtain funding to further investigate arbaclofen.

\section{Disclosure}

The author reports no conflicts of interest in this work.

\section{References}

1. APA. Diagnostic and Statistical Manual of Mental Disorders. 4th ed. Washington, DC: American Psychiatric Association; 1994.

2. Volkmar FR, McPartland JC. From Kanner to DSM-5: autism as an evolving diagnostic concept. Annu Rev Clin Psychol. Epub December 9 , 2013.

3. Kulage KM, Smaldone AM, Cohn EG. How will DSM-5 affect autism diagnosis? A systematic literature review and meta-analysis. J Autism Dev Disord. Epub February 16, 2014.

4. Developmental Disabilities Monitoring Network Surveillance Year 2010 Principal Investigators. Prevalence of autism spectrum disorder among children aged 8 years — autism and developmental disabilities monitoring network, 11 sites, United States, 2010. MWR Surveill Sum. 2014;28 63 Suppl 2:1-21.

5. Autism and Developmental Disabilities Monitoring Network Surveillance Year 2002 Principal Investigators; Centers for Disease Control and Prevention. Prevalence of autism spectrum disorders - autism and developmental disabilities monitoring network, 14 sites, United States, 2002. MMWR Surveill Summ. 2007;56:12-28.

6. Schaefer GB, Mendelsohn NJ; Professional Practice and Guidelines Committee. Clinical genetics evaluation in identifying the etiology of autism spectrum disorders: 2013 guideline revisions. Genet Med. 2013;15(5):399-407.

7. Rossignol DA, Genuis SJ, Frye RE. Environmental toxicants and autism spectrum disorders: a systematic review. Transl Psychiatry. 2014;4:e360

8. Hallmayer J, Cleveland S, Torres A, et al. Genetic heritability and shared environmental factors among twin pairs with autism. Arch Gen Psychiatry. 2011;68(11):1095-1102.

9. Rossignol DA, Frye RE. A review of research trends in physiological abnormalities in autism spectrum disorders: immune dysregulation, inflammation, oxidative stress, mitochondrial dysfunction and environmental toxicant exposures. Mol Psychiatry. 2012;17(4): 389-401.

10. Coghlan S, Horder J, Inkster B, Mendez MA, Murphy DG, Nutt DJ. GABA system dysfunction in autism and related disorders: from synapse to symptoms. Neurosci Biobehav Rev. 2012;36(9):2044-2055.

11. Chadman KK, Guariglia SR, Yoo JH. New directions in the treatment of autism spectrum disorders from animal model research. Expert Opin Drug Discov. 2012;7(5):407-416.
12. Stafstrom CE, Hagerman PJ, Pessah IN. Pathophysiology of Epilepsy in Autism Spectrum Disorders. In: Noebels JL, Avoli M, Rogawski MA, Olsen RW, Delgado-Escueta AV, editors. Jasper's Basic Mechanisms of the Epilepsies. 4th ed. Bethesda, MD: National Center for Biotechnology Information; 2012.

13. Tranfaglia MR. The psychiatric presentation of fragile $\mathrm{x}$ : evolution of the diagnosis and treatment of the psychiatric comorbidities of fragile X syndrome. Dev Neurosci. 2011;33(5):337-348.

14. Clifford S, Dissanayake C, Bui QM, Huggins R, Taylor AK, Loesch DZ. Autism spectrum phenotype in males and females with fragile $\mathrm{X}$ full mutation and premutation. J Autism Dev Disord. 2007;37(4):738-47.

15. Osterweil EK, Krueger DD, Reinhold K, Bear MF. Hypersensitivity to mGluR5 and ERK1/2 leads to excessive protein synthesis in the hippocampus of a mouse model of fragile X syndrome. J Neurosci. 2010;30(46):15616-15627.

16. Yan QJ, Rammal M, Tranfaglia M, Bauchwitz RP. Suppression of two major Fragile X Syndrome mouse model phenotypes by the mGluR5 antagonist MPEP. Neuropharmacology. 2005;49(7):1053-1066.

17. Dolen G, Osterweil E, Rao BS, et al. Correction of fragile X syndrome in mice. Neuron. 2007;56(6):955-962.

18. Carlson GC. Glutamate receptor dysfunction and drug targets across models of autism spectrum disorders. Pharmacol Biochem Behav. 2012;100(4):850-854.

19. Braat S, Kooy RF. Fragile X syndrome neurobiology translates into rational therapy. Drug Discov Today. Epub February 7, 2014.

20. Sanchez-Ponce R, Wang LQ, Lu W, von Hehn J, Cherubini M, Rush R. Metabolic and pharmacokinetic differentiation of STX209 and racemic baclofen in humans. Metabolites. 2012;2:596-613.

21. Lal R, Sukbuntherng J, Tai EH, et al. Arbaclofen placarbil, a novel R-baclofen prodrug: improved absorption, distribution, metabolism, and elimination properties compared with R-baclofen. J Pharmacol Exp Ther. 2009;330(3):911-921.

22. Vakil NB, Huff FJ, Bian A, Jones DS, Stamler D. Arbaclofen placarbil in GERD: a randomized, double-blind, placebo-controlled study. Am J Gastroenterol. 2011;106(8):1427-1438.

23. Gerson LB, Huff FJ, Hila A, et al. Arbaclofen placarbil decreases postprandial reflux in patients with gastroesophageal reflux disease. Am J Gastroenterol. 2010;105(6):1266-1275.

24. Vakil NB, Huff FJ, Cundy KC. Randomised clinical trial: arbaclofen placarbil in gastro-oesophageal reflux disease - insights into study design for transient lower sphincter relaxation inhibitors. Aliment Pharmacol Ther. 2013;38(2):107-117.

25. Nance PW, Huff FJ, Martinez-Arizala A, et al. Efficacy and safety study of arbaclofen placarbil in patients with spasticity due to spinal cord injury. Spinal Cord. 2011;49(9):974-90.

26. Howick J, Chalmers I, Glasziou P, et al. The Oxford 2011 Levels of Evidence: Oxford Centre for Evidence-Based Medicine; 2011 [cited September 3, 2011]. Available from: http://www.cebm.net/index. aspx?o=5653. Accessed March 21, 2014.

27. Henderson C, Wijetunge L, Kinoshita MN, et al. Reversal of diseaserelated pathologies in the fragile $\mathrm{X}$ mouse model by selective activation of GABAB receptors with arbaclofen. Sci Transl Med. 2012;4(152): $152 \mathrm{ra} 28$.

28. Pacey LK, Tharmalingam S, Hampson DR. Subchronic administration and combination metabotropic glutamate and GABAB receptor drug therapy in fragile X syndrome. J Pharmacol Exp Ther. 2011;338(3): 897-905.

29. Berry-Kravis EM, Hessl D, Rathmell B, et al. Effects of STX209 (arbaclofen) on neurobehavioral function in children and adults with fragile X syndrome: a randomized, controlled, phase 2 trial. Sci Transl Med. 2012;4(152):152ra27.

30. Erickson CA, Veenstra-Vanderweele JM, Melmed RD, et al. STX209 (Arbaclofen) for autism spectrum disorders: an 8-week open-label study. J Autism Dev Disord. 2013;44(4):958-964.

31. Delahunty C, Walton-Bowen K, Kuriyama N, et al. Randomized, Controlled, Phase 2 Trial of STX209 (Arbaclofen) for Social Function in ASD. Orlando, FL: American Acadamy of Pediatrics; 2013. 
Drug, Healthcare and Patient Safety

Dovepress

\section{Publish your work in this journal}

Drug, Healthcare and Patient Safety is an international, peer-reviewed open-access journal exploring patient safety issues in the healthcare continuum from diagnostic and screening interventions through to treatment, drug therapy and surgery. The journal is characterized by the rapid reporting of reviews, original research, clinical, epidemiological and

post-marketing surveillance studies, risk management, health literacy and educational programs across all areas of healthcare delivery. The manuscript management system is completely online and includes a very quick and fair peer-review system. Visit http://www.dovepress.com/ testimonials.php to read real quotes from published authors.

Submit your manuscript here: http://www.dovepress.com/drug-healthcare-and-patient-safety-journal 